\title{
OBSERVATIONS ON THE AËRIAL TRANSMISSION OF THE ENTERIC FEVER POISON,
}

\author{
With a Record of an Outbreak presumably caused by that Means \\ of Infeetion.* \\ BY JOHN BROWNLEE, M.A., M.D., D.P.H., \\ Pupil Assistant, Glasgow Sanitary Office.
}

There are few problems which have so often seemed near solution, but of which the solution has been found on examination to be incomplete, as that of the means by which enteric fever is spread. The research has been carried on by two different methods, roughly divided into two periods by the year 1880, which marks the discovery of the bacillus by Eberth. Before that date the investigation was confined to the observation of the conditions and circumstances under which enteric fever arose, spread, and diminished, and there was a general consensus of opinion that the presence of putrefying filth, which infected either directly or by means of the pollution of water or milk, was essential to its production. But opinions were divided as to whether filth of itself could acquire the specific property which engendered the disease, or whether the addition of some definite infective material was necessary to render it dangerous. All this now is but the echo of the famous controversy on spontaneous generation. This doubt was finally, though not immediately, set at rest by the discovery of the enteric bacillus. But the new discovery brought new difficulties. Laboratory experiment decided the life of the organism to be precarious, that there could be no doubt that it might live in water for a short time, say about a fortnight, but that it speedily disappears, and does not multiply ; and that, mixed with other organisms, it quickly perishes in the race for existence. It was supposed that it could not be recovered from infected soil, or even from the sewage of a fever hospital, into the drains of which the discharges of enteric fever patients had been thrown without disinfection. In fact, the result of all the experiments seemed to show that it was a wonder that the bacillus had not long since disappeared from the earth. Within the last two years the great facts, which were the basis of the former theories, have recurred so often and made themselves so prominent, that re-investigations of the characteristics of the micro-organism have been made under a new variety of conditions. These experiments have been rendered much more easy

* Read before the Philosophical Society of Glasgow, April, 1898. 
by the discovery of new means of isolating and identifying the organism.

To begin with, I shall give a brief account of what has been done by the various observers, and thereafter discuss the general state of knowledge of the subject at the present time.

It has been found, in the first place, that if ordinary arable land be infected with the bacillus, the latter can show a prolonged vitality. Dr. Robertson,* Medical Officer of Health of Sheffield, has recently published an account of some interesting experiments begun in July, 1895. He had the turf carefully removed from the surface of several patches of an ordinary grass field. These patches of ground were watered with broth-cultures of the enteric bacillus diluted in water, in one on the surface, in another at 9 inches depth, and in a third at 18 inches depth. In the latter cases the soil was first removed to the depth mentioned in the order of its stratification, and then carefully replaced. During the autumn, 130 days later, he found on examination, not only that the bacilli which were sown on the surface had multiplied, but even that those which were sown 18 inches beneath the surface had so proliferated that the bacillus could now be found in the surface layer of the soil. Again, he found during the cold months of winter that from these and other patches of ground which had been similarly treated in the autumn no enteric bacilli were recoverable. During the spring some of the patches were watered with very dilute sterile broth, to imitate in a measure the continuous contamination of the soil which may be supposed to take place in certain localities by sewage. It was found that from those which were so treated the bacillus could in June be recovered with ease, but none at all could be obtained from the patches which were not so treated with dilute broth. It would thus seem that, though apparently absent in the cold of winter, the bacilli might be nursed back in the warmer weather to an active existence if the means of subsistence were supplied. It would have added interest to the experiment if those patches of soil in which the bacilli were not detected in June had then been watered with broth, as the others were, to discover whether, even after that longer interval of time, a further growth of the bacilli could have then been induced.

In association with these must be narrated the experiments of Dr. Sidney Martin. $\dagger$ In those, however, the natural conditions were not imitated so closely as in those of Dr. Robertson, and they are therefore not so conclusive. Dr. Martin obtained numerous

* British Medical Journal, January 8th, 1898.

+ Local Government Board Reports, 1896-97. 
specimens of soil, some from sources grossly polluted, contaminated with organic matter from localities in which enteric fever was endemic. Besides these he procured specimens of virgin soil from the downs of Hampshire. These were chosen from the deeper layers which underlie the surface peat, and consist of a mixture of sand and completely decayed vegetable matter. Part of each of these samples was pulverized, placed in an Erlenmeyer's flask, moistened, and then sterilized. It was found that the growth of the bacillus was rapid and luxuriant in the polluted earth, especially at higher temperatures, $90^{\circ}$ to $100^{\circ} \mathrm{F}$, and though much slower at the temperature of the room, that even then it took place. In these flasks the bacilli were still active after the lapse of 105 days, the duration of the experiment at the time the results were published. With regard to the virgin earth a much different result appeared. Not only did proliferation not take place, but even after a time the living bacillus could not be obtained at all. It seemed completely killed out.

It would seem, then, that these two observers agree in their opinion that, given suitable conditions, the bacilli of enteric fever may retain its vitality in the soil for a lengthened period. But what are those suitable conditions? Sewage as a suitable medium for the growth of the bacillus had been set aside by former observers, and a repetition of the experiments on this point by Dr. Klein* resulted in their confirmation. But this observer, in view of the changes which occur in the natural purification of sewage, of which the most notable is the formation of nitrates by oxidation, tried whether the artificial addition of these salts to crude sewage would produce a more suitable medium. He discovered that the bacillus does not die out rapidly, as was observed in former experiments, but grows luxuriantly, the addition of nitrates converting the sewage from an unsuitable medium into. one very favourable to their growth.

In another series of experiments he found that if the bacillus were added to certain of the London drinking waters, it could be found after the lapse of five to eight weeks, and in certain lake waters even longer. This is a much longer period than had been thought of. Frankland found that in Loch Katrine water, which was apparently exceptionally favourable, the duration of life was. but twenty-five days. The results of these experiments apparently show that, while the pollution of water directly by sewage might not be of serious moment for more than a short time, yet if the character of the medium intervening between the source of pollution

* Local Govermment Board Reports, 1894-95. 
and the point at which the water is taken for use be of such a nature as will admit of the ready formation of nitrates, on which the growth of the bacillus seems largely to depend, then specific contamination may continue for a lengthened period of time.

There are other points, however, in the condition of its life. Some media which are unsuitable for the growth of the enteric bacillus can be made suitable by the action of other micro-organisms; for instance, as has been noticed already, the action of the nitrifying bacilli make sewage a suitable medium. Pure gelatine is not a suitable culture medium, yet in some experiments made by myself it was found that if liquefied by certain other micro-organisms, the resulting fluid was most suitable. This, however, was not a constant result with different species of liquefying organisms. In a few other experiments, made with a view to ascertaining where the typhoid bacillus can maintain itself under natural conditions, several specimens of soil were chosen. These consisted of peat which had overgrown a rocky patch in an open moor, and beyond suspicion of contamination by manure and sewage, decayed leaves from a wood, and the detritus from the face of a weathered trap rock. These samples of earth matter were extracted with water. The resulting fluids were placed in tubes and sterilized in the usual manner, and then inoculated with the enteric bacillus. It was found that in the extracts from the peat and the leaves the bacilli had disappeared after ten days, while they grew abundantly in the extract prepared from the weathered rock. This result was probably due to the fact that the detritus was gathered from a locality which was exposed, to a slight extent, to surface water from a manured field. The medium composed of decayed vegetable matter only did not afford anything like so suitable a medium as that contaminated by even a trace of animal organic matter.

I have already referred to the spread of enteric fever by polluted water or milk, but there is in addition another possible means of infection, namely, the aërial dispersion of the organisms. The most natural means, the dispersion of soil, is in the form of dust, when it is easily carried about by the wind. It is well known that the bacillus of enteric fever does not readily survive complete desiccation. The limit of the duration of its life in this state Flügge, from his own experiments, in which the bacilli were dried in thin layers, has given at five to fifteen days. By Germano,* in a paper published last August, no certain limit is given, as in his opinion

* Zeitschrift fiur Hygiene, chap. xxiv., p. 3, reported in Public Health, ix. 360 (August, 1897). 
this limit depends on the amount of drying to which the bacilli are subjected. Half dried, they lived for some months; wholly dried, for but a short time: He found that on garments of wool the infection lingered longer than on those of cotton, a fact which he explains by the greater power the former has of retaining moisture. Now, ordinary soils possess the property of retaining in their interstices a certain proportion of moisture, and do not yield this to the ordinary natural means of drying, such as currents of air. In a few experiments made by myself in this connection, ordinery potting soil was placed in a porcelain dish, and thoroughly dried in a hot-air sterilizer. It was further subjected to continuous dry heat long enough to kill any micro-organisms present. This soil was then moistened with a broth culture of the enteric bacillus. By this means it was practically assured that the minute passages of the soil, filled by the action of capillarity with the broth, would at the same time be mechanically charged with enteric bacilli to the extent that where there was moisture there would also be the micro-organisms. This porcelain dish was then covered with a glass lid, the whole kept at $98^{\circ} \mathrm{F}$. for twenty-four hours, after which the cover was removed and spontaneous evaporation allowed to take place. In the course of a week this soil had become dry enough to be easily seattered by the breath. It had become, in fact, dust such as can be readily blown about by the wind. As it might be assumed with certainty that the soil with which the experiment was conducted had become contaminated by microorganisms derived from the surrounding air, it was now saturated with meat broth, to which a little carbolic acid had been added, the presence of the latter restricting the growth of those other microorganisms while allowing the free growth of the enteric bacillus. Plates of Elsner's potato gelatine were made from the earth so treated, and a bacillus recovered which agreed in its more important characteristics, microscopical and others, with the enteric bacillus. A similar experiment was made with unsterilized soil, but the colonies of the Bacillus coli communis were so numerous that it was found impossible to separate the enteric bacillus from these, owing to the close resemblance in character of the two organisms. This experiment was undertaken solely with a view to decide whether the aërial transmission of the bacillus be possible, and was consequently carried out under conditions which favoured that micro-organism as much as possible. The fact that an active bacillus was recovered after almost complete drying, when the soil was in a condition capable of being carried about by the wind, indicates at least the possibility of aerial transmission. The extent 
to which this takes place can only be decided by an appeal to natural processes, and not in the laboratory.

Having thus briefly explained the present state of experimental knowledge regarding the enteric bacillus, before passing to an account of the outbreak, of which the present paper is a record, I would wish to make a few remarks concerning the conditions under which the enteric microbe conducts itself in Nature's laboratory, or, in other words, the manner in which enteric fever rises, spreads, and disappears. A seasonal undulation has been found to exist showing a maximum and minimum range. Not only in endemic prevalence of this fever, but also in the occurrence of the epidemic form of the disease, due to pollution of water and milk, this is well marked. Of 200 water-borne epidemies of enteric deseribed by Mr. Hart, 40 per cent. began in the months of July, August, or September. This, however, is of interest only as a rough approximation. But if we consider the more accurate means of information provided by the number of notifications per month in different centres of population, more definite results may be arrived at. It is found, for example, that in Glasgow the second and third weeks of August may be counted upon to produce from 80 to 100 new cases of enteric fever. This rate increases through the whole of September, and then begins to decline. But the decline is much slower than the rise. If the winter be mild, the minimum may not be reached till the beginning of April of the following year. On the other hand, with a cold and frosty winter it may be reached by the end of January. Further, a cold spring and summer may delay the onset of the fever till a later period in the autumn. In London, on the other hand, where the summer is warmer and the winter colder, we have a somewhat different state of affairs. The numbers by notification begin to Increase earlier in the year (July), and continue to increase till the month of October, after which the fall is rapid. The colder winter seems to ensure that the spring will be free from the disease, both absolutely and also relatively to Glasgow. In India, in the province of Bengal, we find enteric fever most prevalent during the hottest season of the year (May, June, and July). From these facts it would seem that the virulence of the organism producing the fever is cumulative, or, in other words, is built up gradually. This maximum of virulence, once reached, is apparently retained for a considerable period, and diminishes slowly. It may be destroyed by cold, as is indicated by $\mathrm{Dr}$. Robertson's experiments before referred to. It may, in the absence of severe cold, last even through the winter; but the decrease once initiated is arrested only after a considerable time, even although 
the surroundings appear favourable. Conditions favourable to the organism may, however, exist even in winter, resulting in great epidemics. If the bacillus exist in filth, especially under snow, a sudden thaw and flood washing the filth into a water-supply may cause a deadly epidemic. Organic filth is a fermentable substance, and can maintain a comparatively high temperature for a considerable time after that of its surroundings has fallen even below freezing-point. A non-conductor such as snow would probably favour this, and a condition is thus produced which may be presumed to aid the growth of the bacillus. It was under such a condition the great epidemic in the Tees Valley, in Darlington and Middlesbrough, in the winter of 1890-91, and also the recent outbrealk at King's Lynn, took place. In both cases much accumulated filth was washed into the sources of drinking-water by heavy floods, and in both an epidemic was produced at a season of the year at which it is not usually looked for. It might be argued, however, that a disease which is due to infection of the digestive track may have an early autumnal seasonal prevalence from causes other than the more potent properties of the bacillus. This season also coincides with a large increase in diarrhoeal disorders from the dietetic changes corresponding to it. But the fact that food epidemics, such as those due to pollution of milk, spread with equal virulence whether they occur in winter, spring, or autumn (the most severe of such epidemics in Glasgow was at the end of April), shows that it is the bacillus which requires for its potency a favourable environment, and that, granted such, there is no need for its host to be enervated by summer heat or predisposed by seasonal unsuitability of food.

From the fact already stated, that the virulence of the enteric bacillus is so slowly lost, we are led to infer-what is a matter of general experience-that an epidemic of typhoid fever in a filthy locality, albeit sudden in onset, has a slow wane. The typhoid bacillus having found a resting-place, has, it is true, a number to its days if suitable nourishment be not supplied to it; but it unfortunately only too often happens that the same carelessness which gives it a foothold also affords it a continual supply of nourishment. As an instance of this longevity of the bacillus may be mentioned a curious incident in an epidemic at Coalville Packington, in Leicestershire. One well which had been instrumental in the spread of the disease was re-opened thirteen weeks after it had been closed. The locking up of this well was followed by an immediate cessation of new cases, while its re-opening was followed by a fresh ontbreak. 
Further evidence, pointing in the same direction, is given by Dr. Childs* in a paper dealing with the history of enteric fever in Munich. This eity, which is now almost ideally free from the fever, was formerly one of the plague spots in Europe. Of every 100,000 of its inhabitants between 200 and 300 died every year from typhoid fever. This number of deaths probably represents ten times the number of cases, for in towns where the fever is endemic the victims are largely children, and the mortality consequently low. The conditions which favoured this prevalence in Munich were the following:

The eity is built upon a mixed surface soil, beneath which is a bed of gravel of varying depths, which in its turn rests on a bed of impervious marl. Through this gravel bed filters a constant horizontal stream of water, which flows parallel to the River Iser and beneath the eity. This underground water was conveniently used both to get rid of the sewage and also as the source of the water-supply. Cesspools which were loosely constructed received the drainage; the water for domestic use was obtained from shallow wells. The slaughter-houses, of which there were a great number in the city at one time, had no other drainage. The more fluid refuse from these was likewise constantly allowed to pollute this source of water-supply. Improvements were gradually introduced. The cesspools were rendered water-tight, and this alone was followed by a decrease in the enteric death-rate. A better watersupply was introduced, and, lastly, the slaughter-houses were all abolished in the year 1878. This last act was followed by a great and noticeable decrease in the number of deaths from enteric fever. This sudden improvement is rendered more interesting when we remember that serum albumen, such as must have constituted a fair proportion of the soakage from the slaughter-houses, is one of the most suitable nourishing media for many disease-producing organisms. The general conclusions arrived at by Dr. Childs are as follows: (1) The drinking-water has not played an important part in producing and reducing the typhoid epidemics of Munich; (2) The great prevalence of typhoid fever in Munich was due to the great pollution of the soil (including specific pollution), modified by certain unknown conditions in the soil which are correlated with the movements of the subsoil water; and (3) The gradual reduction of the typhoid fever was due to the gradual purification of the soil, and the almost abrupt termination of the epidemics to the sudden removal of all the slaughter-houses. He further suggests that the

\footnotetext{
* "Transactions of the Epidemiological Society," 1897-98. See also Public Health, x. 169 (February, 1898).
} 
character of the soil may be a very important factor in the production of an epidemic.

The conclusion that the drinking-water, under certain circumstances, has little to do with the endemic nature of the fever has a great interest. Much the same conclusion has been arrived at by Dr. Bulstrode* regarding the prevalence of enteric fever in Chichester, which has geological conditions almost exactly the same as those of Munich, and which had a like regard for convenience and disregard for sanitation. In both these towns the fever seems to have attacked about equally those who drank pure and impure water. Of course, we must very much hesitate to accept this conclusion without question, for from the inter-association of people there is naturally a great deal of difficulty in absolutely deciding that a given individual has not drunk the contaminated water.

But even with an irreproachable water-supply, contaminated soil alone may give rise to the continued presence of enteric fever. In the burgh of Rntherglen, $\uparrow$ for instance, for a number of years, in the older part of the town, there has been a notable disregard for modern sanitation, no water-closets, untrapped sinks, ashpits below the level of soil, with drop-privies, and unpaved back-courts being the rule. Yet, notwithstanding, there was no undue prevalence of enteric fever prior to the year 1894. During the autumn of that year a dairy in the district became a centre of infection, and certain of the streets in this part of the town were invaded. Since then enteric fever has been constantly present.

During 1895 the attack-rate in this part of the town was ten times, during 1896 three times, and during 1897 four times that of the rest of the burgh. Specific soil-pollution seems the only probable source of the infection. For it is to be noted that other streets, differing in no respect from these in point of sanitation except as regarding the absence of primary infection, have not been so attacked.+ +

* Local Government Board Reports, 1896-97.

† For the information here given $I$ am indebted to the kindness of Dr. Buchanan, Medical Officer of Health of the burgh.

\pm As a further evidence of the manner in which polluted soil may spread infection, there is here mentioned a series of cases which occurred in al single tenement of houses in Glasgow. Of this tenement the sole water-supply is that of the city of Glasgow, viz., from Loch Katrine. The condition of this tenement was, as regards sanitary matters, very unsatisfactory, the drains especially being very defective, yet no cases of enteric fever had occurred there for a number of years until one was imported in September, 1893. Of the subsequent cases no two occurred in the same house. This fact, and the distance of time between the successive cases, would seem to show that the infection was indirect, i.e., from the soil, and not from patient to patient. 
The conditions for continuous soil-pollution are here seen at their best. For as enteric fever is a disease of gradual onset, there is little doubt that each new case as it occurs will lead to the deposition of infected matter in the ashpits, which ceases only when the illness has become severe enough to demand medical attendance. The refuse from these ashpits is first emptied out into the street and thence removed. This method of removal produces fouling of the street; a certain amount of refuse, being left behind, is converted into dust and scattered about by the wind. We have but to look upon a basin of milk which has stood uncovered for a single night to see how much dust is deposited from the atmosphere, and if that dust be specifically polluted, there is no end to the vicious circle which keeps up both the supply of the poison and its distribution. That this is not purely theoretical is seen from the fact that Professor Delépine,* of Manchester, has obtained the enteric bacillus from the soil lying between the bricks forming the floor of an ashpit. In this case the contamination had taken place at least two months previously, and probably as far back as thirteen months. Either period, however, allows sufficient margin for the spread of the fever by the means under discussion.

The following series of cases which occurred in the City of Glasgow Fever Hospital, Kennedy Street, will furnish a clear example of the manner in which soil may be the means of aiding the spread of the fever. This hospital consists, as may be seen from the diagram, of seven pavilions of two wards each. The three northern pavilions, with the nurseg' home, are new, and have a separate drainage system which discharges into North Oswald Street, while the drains of the southern wards, together with those of the kitchens and administrative block, discharge into the main sewer in Kennedy Street. The ramifications of the drains will be appreciated more easily by a glance at the plan than by any description. The whole drainage system of the northern wards

Table of Sequence of Events.

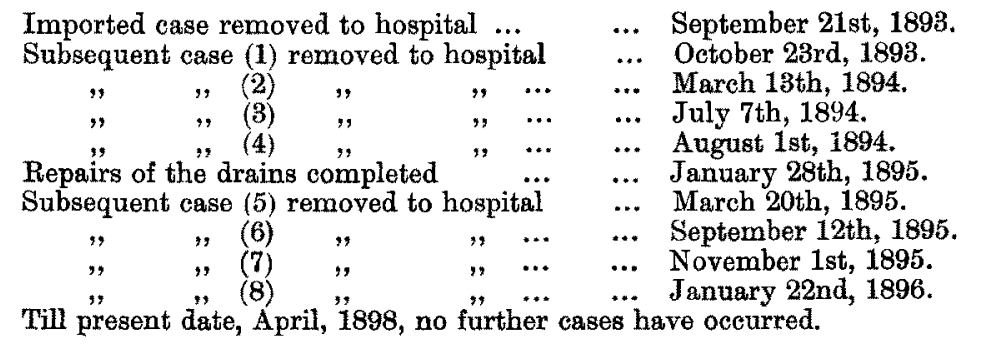

* Public Health, x. 154 (February, 1898). 

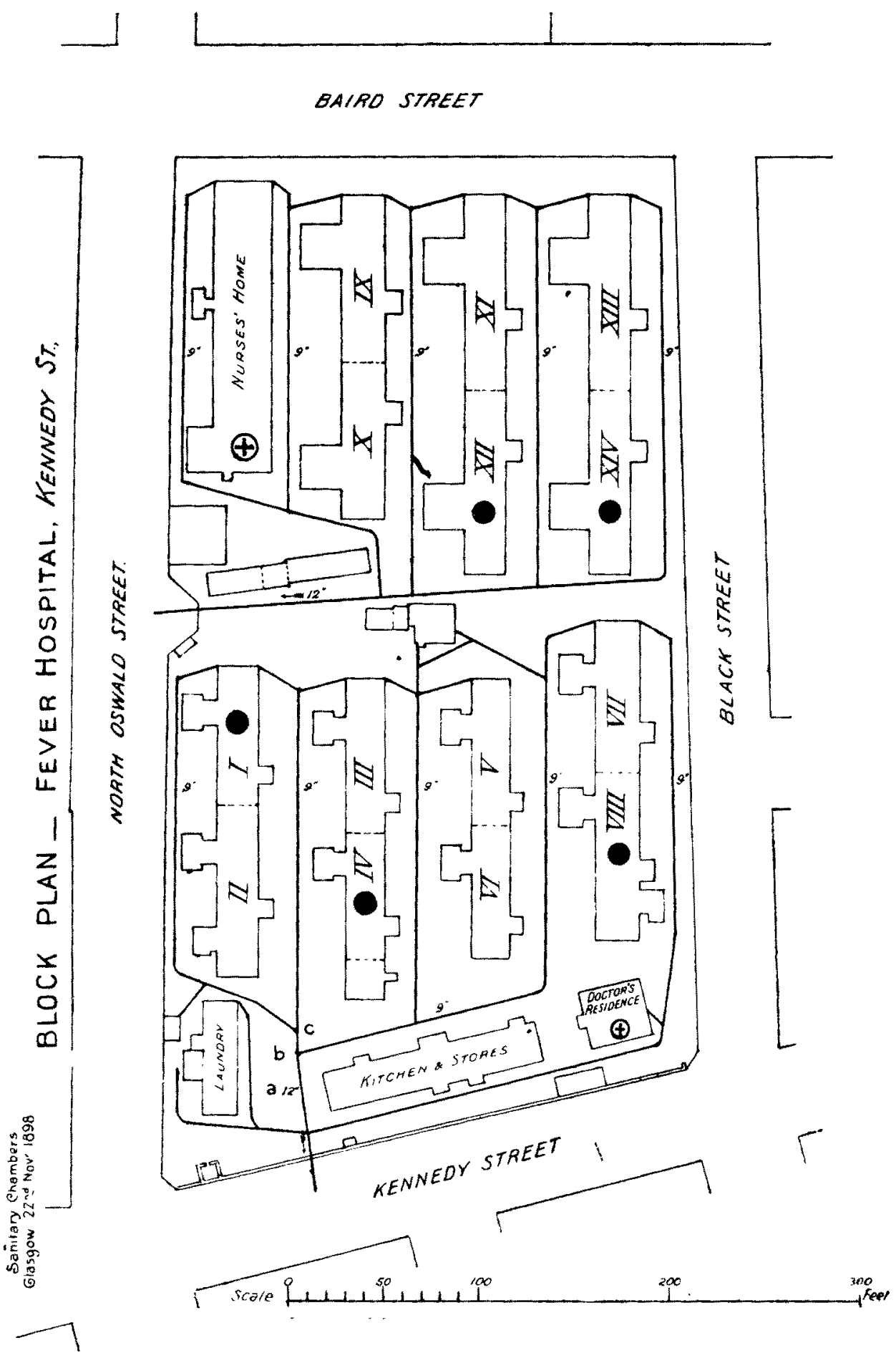
was constructed about four years ago, and, when tested after the outbreak, was found in almost perfect order. With regard to that of the southern and older wards, it may be stated that when the hospital was re-opened a few years ago it was thought sufficient to renew the fittings in more immediate connection with the wards alone. The water-closets, baths, traps, and ventilating-shafts were renewed, but it was not thought necessary to relay the deeper part of the drainage system, it being considered at the time that the necessity for the hospital would be but temporary.

Owing to the absence of a grease-trap, the main effluent drain from the southern wards became gradually choked, till ultimately no flow of sewage was possible along the pipes. Under the resulting pressure the lutings of the pipes gave way and free leakage ensued. As a result, subsidence of the soil, which was of a porous nature, occurred, so that intermittent saturation of the subsoil with sewage was possible. The porosity of this subsoil, which was composed largely of furnace ashes, cinders, pottery rubbish, etc., while undoubtedly of great advantage in affording special facilities for natural purification, yet, according to the paper by Dr. Klein already referred to, would seem in this case to have rendered possible a prolonged existence of the enteric bacillus. The defects of the drains were discovered early in April, and their reconstruction was immediately entered upon. In the ordinary course of events this would probably not have had any serious effect, but in this instance several enteric patients had been inmates of the hospital, and their discharges were passed into the defective drains. These patients had been certified as scarlatina, but on admission no . evidence of this disease could be discovered, and further observation showed them to be suffering from enteric fever. The first, a child of three, was admitted on December 31st, 1896, and by the time the nature of the case was sufficiently defined she was too ill to remove to Belvidere. This child was convalescent by the end of January. The second was a girl of fourteen years of age, who was admitted on January 12th, 1897, and transferred to Belvidere on January 14th, while the third took ill on February 19th, was recognised as enteric fever on February 26th, and transferred next day. Thus from December 31st till February 27th there was a more or less constant stream of infected dejecta passing into the drains. Of course, the ordinary means were taken to disinfect the discharges, but it is well known that these, however thoroughly applied, are of doubtful efficiency. All these patients were in the same ward, viz., Ward III. (the observation ward), whither they were sent on admission, and where they remained till dismissal 
from the hospital. It will thus be seen that the southern drainage system alone was specifically infected.

From this time there was no other case of enteric fever in the hospital till the indigenous cases occurred. Repairs of the drains were begun at the main effuent sewer about April 10th, and the obstruction removed. On May 6th a patient in Ward VIII. (southern section) had an evening rise of temperature, which was the first indication of what subsequently proved to be an attack of enteric fever. This patient was a boy of nine years of, age, who had been five weeks in the hospital, and who (owing to scarlatina complications) had never been out of bed. On the fifth day, from the rise of temperature just referred to, the diagnosis was fairly certain. Owing to the drains being exposed lower down, great care was taken in disinfecting his dejecta. These were thoroughly mixed with crude carbolic acid, and allowed to stand a considerable time before being put into the drains.

On the suspicion of enteric fever arising, plate cultivations were made from the soil at the part where the men were at that time at work (marked $b$ on the plan). The earth examined was taken from a part some inches above the level of the drain, so that the possibility of contamination from the motions of this last case might be as far as possible avoided. From this soil a micro-organism was obtained which, in several of the most important features, completely agreed with the typhoid bacillus.* Cultivations were also made from the soil midway between the surface and the drain (i.e., about $2 \frac{1}{2}$ feet below the surface), with negative result as regards the typhoid bacillus. Search for the same bacillus was now made in some of the soil, thrown up in the process of excavation, which had dried in the sun. Though in laboratory experiments the enteric bacillus is easily killed by drying, yet dust, as already stated, even on the hottest day, contains a certain amount of moisture. Plates were made with Elsner's potato gelatine, and an actively mobile non-liquefying bacillus obtained, which in a gelatine stab-culture formed no gas bubbles, and which gave a characteristic growth on an agar streak-culture. Unfortunately, when the further investigation of the properties of the bacillus was resumed, it was found that the culture had lost its vitality. This bacillus, which was probably the enteric bacillus, was recovered from soil which could not have been contaminated by the dejecta of the case then in hospital.

Cultivations were also attempted on gelatine plates with the grease from the choked drain, but no organism resembling the Bacillus coli communis or the enteric bacillus could be found.

\footnotetext{
* Characteristic growth on agar and potato; non-formation of bubbles in a gelatine stab-culture; Widal reaction.
} 
From May 6th, when the first patient exhibited signs of enteric fever, till June 30th, no further cases occurred; then they followed in quick succession, as will be seen by a reference to the accompanying table:

Table of alt the Indigenovs Cases in Hospital.

\begin{tabular}{lrrrrlllllr} 
& \multicolumn{3}{c}{ Sex. } & \multicolumn{2}{c}{ Age. } & \multicolumn{3}{c}{ Admitted. } & \multicolumn{3}{c}{ Showed First Bigns. } & Ward, \\
J. C. & $\ldots$ & M. & $\ldots$ & 9 & $\ldots$ & April 2nd & $\ldots$ & May 6 th & $\ldots$ & VIII, \\
P. H. & $\ldots$ & F. & $\ldots$ & 2 & $\ldots$ & May 7th & $\ldots$ & June 30th & $\ldots$ & I. \\
S. M. & $\ldots$ & F. & $\ldots$ & 22 & $\ldots$ & May 16th & $\ldots$ & July 1st & $\ldots$ & XIV. \\
J. K. & $\ldots$ & F. & $\ldots$ & 7 & $\ldots$ & June 29 th & $\ldots$ & July 14th & $\ldots$ & IV. \\
H. W. & $\ldots$ & M. & $\ldots$ & 10 & $\ldots$ & July 9 th & $\ldots$ & July 21st & $\ldots$ & XII.
\end{tabular}

Clinically, the features in each case were those of mild enteric fever, except P. H., in the case of whom the symptoms were somewhat anomalous. At the onset these were of a severe type, but subsided rapidly. She, however, gave a most typical Widal reaction. The circumstances which preceded the first case of enteric have already been detailed, and I shall now proceed to explain the further repairs carried out upon the drainage system at the various periods during the continuance of the enteric cases in the hospital. That portion of the drains below $a$ and $b$ (see plan) was the first portion taken up and relaid, and immediately thereafter the drain connections between this and the laundry, kitchen, and administration block were renewed. This work occupied the whole of May, and by the second week of June further work was commenced on that portion of the main drain marked $b$ to $c$ (see plan), which, as will be seen, was exposed both to contamination from the original imported cases as well as of the first indigenous case. Two patients were now attacked by the disease - a young woman and a child. Two and three weeks later respectively, two other cases of enteric fever occurred in the hospital. Those patients were both children, aged seven and ten, who had been strictly confined to bed from the day of admission. Both were mild but very characteristic cases of enteric fever. Of these cases two were resident in the northern and two in the southern portion of the hospital.

Outbreaks of enteric fever are usually associated with cases of slight febrile derangement, which cannot be definitely diagnosed as enteric fever. A resident physician and a nurse were thus affected.

So far as could be judged from the history of the patients and their environment before admission to hospital, there was no reason to suppose that any of them were incubating enteric fever on admission, and this opinion was further borne out by the length of time which elapsed in nearly all the cases between date of admission 
and the first onset of symptoms (see table). With regard to sources of infection within the hospital precincts itself, these fall to be considered :

1. The Milk Supply.-This was the same as that of the City of Glasgow Fever and Small-pox Hospitals, Belvidere, where no indigenous cases of enteric fever occurred at this time.

2. Defective Drains and Traps connected uith Tuo Infected Wards. -This source of infection is rendered very unlikely from the facts (a) that careful smoke-testing revealed no practical defects in the traps or soil-pipes connected with the wards; $(b)$ that wards connected with both southern and northern drainage systems were infected, although, as already explained, the northern system of drainage was not in any way contaminated by the infected discharges from patients till the time when the first case occurred in Ward XII.; $(c)$ the enteric poison (when we remember the date of admission of the first enteric case) was present in the drains of the southern section four months before the occurrence of the first indigenous case.

3. The Soil turned up during the Repairs carried out on the Drains. -(a) The first indigenous case occurred in hospital about three weeks after the upturning of the soil when repairs were undertaken on that portion of the main drains marked $a-b$, while the second group of cases occurred from three to five weeks after that, when the portion marked $b-c$ was taken up; (b) the sudden cessation of the indigenous infection as soon as the repairs to the main drains were completed; (c) the two cases which arose in the northern section of the hospital must have occurred as the result of some form of aerrial infection, in view of the fact that the northern and southern drainage systems had no communication with each other; $(d)$ that this source of infection was possible, and even highly probable, is manifest from the fact that micro-organisms presenting most of the characteristics of the enteric bacillus were procured from the upturned soil, which, owing to the warmth of the weather at the time, became rapidly dry, and so was readily carried about by the wind.

That this outbreak was most probably the result of infection present within the hospital precincts is shown by the manner in which it died out, as already described, when at the same time an outbreak of enteric fever present in the city was increasing steadily in extent and severity.

The proximity of the kitchen and stores to the turned-up soil during the repairs carried out on the drainage system makes it at least possible that the patients were infected by the food, which 
had in the first instance been contaminated by dust containing the specific organism.

As regards, then, the aërial spread of enteric fever, the conclusions which are of importance are as follows :

1. More especially in large towns, all back-courts should be provided with an impervious pavement. Earth or causewayed courts are open to organic fouling, especially by children. This fouling on an asphalted court is of less moment, as absorption cannot take place.

2. Such back-courts ought to be carefully scavenged daily, or oftener in enteric districts, with a plentiful use of water.

3. Wet ashpits are extremely objectionable, and should not be tolerated, for not only do they provide a fertile soil for the propagation of the bacillus, but their cleansing may be a new source of the disease, both by aërial contamination, as already indicated, and also by the conveyance of the bacillus to new and possibly as yet unpolluted localities.

4. From what has already been said, it will be evident that a series of sporadic cases might readily occur in a district without any apparent connection among themselves, yet which, if our knowledge of the train of associated circumstances were complete, might be traced back to a common source of infection.

Supposing, for example, that the earth from a court contaminated with the enteric poison were turned up during the prevalence of dry weather, it might easily happen that the dust carried about by currents of air and deposited on milk, potted meat, bread, cheese, or other articles of food usually consumed without further cooking, might be the true source of infection. The difficulty would be further increased by the fact that, before these sporadic cases could come under observation, the actual source of infection would have been long obliterated.

Sewage Schemes.- The Town Council of Andover and the Urban District Council of St. Thomas have adopted the "septic tank" system of sewage disposal, with the approval of the Local Government Board.

OBItUARY.-The death of Colonel Waring, of New York., U.S.A., the well-known expert on scavenging and refuse disposal, was announced during the past month. Colonel Waring had been to Cuba to advise as to the cleansing of the capital, and on his return home was taken ill with what proved to be yellow fever. The body was buried at sea, and his house most rigorously disinfected. Colonel Waring's death in the discbarge of duty has been very generally deplored. 